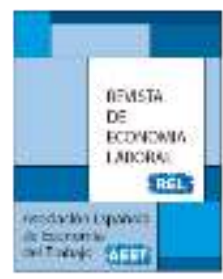

\title{
MONOGRÁFICO "MUJER Y MERCADO DE TRABAJO"
}

\author{
Inmaculada Cebrián López*, Raquel Llorente Heras\& \\ *Universidad de Alcalá, \&Universidad Autónoma de Madrid
}

\section{Presentación}

A lo largo de esta obra se pueden encontrar diferentes visiones que explican las consecuencias que ha tenido para la sociedad, y en particular para las mujeres, los cambios producidos en el mundo de las relaciones laborales desde que, en la segunda mitad de los años 80, se iniciase el proceso de incorporación de las mujeres al mercado de trabajo de manera permanente y sin precedentes en la historia de nuestro país.

Los datos de la Encuesta de Población Activa (EPA) referidos a la participación y el empleo ponen de manifiesto que si bien, en el año 1987, la tasa de actividad de las mujeres era del 32 por ciento, a finales de 2017 era ya del 53 por ciento sobre el conjunto total de la población en edad de trabajar. Este aumento se debe a que las mujeres activas han pasado de ser algo menos de 5 millones a algo más de 10 millones. No obstante, la tasa de actividad de los hombres sigue siendo mayor que la de las mujeres, aunque en algunos grupos de edad, sobre todo entre los más jóvenes, las diferencias prácticamente no existen y con el tiempo la convergencia entre las tasas de actividad de ambos se puede llegar a producir. Ahora bien, esta tendencia ha traído consigo cambios notables que han de ser analizados y valorados por sus consecuencias, unas veces beneficiosas y otras no tanto.

Durante todos estos años el empleo total también ha aumentado, sobre todo el femenino, hasta tal punto que de los 7 millones de personas que entraron en el empleo, 5 millones fueron mujeres. A pesar de todo, la tasa de empleo de los hombres (54,9 por ciento) es superior a la de las mujeres (43,5 por ciento), con diferencias significativas en función de la situación familiar y la presencia o no de hijos en el hogar. Como ejemplo, en 2017, la tasa de empleo de las mujeres entre 30 y 34 años era del 90 por ciento mientras que si había algún hijo menor de 3

\footnotetext{
(C) Revista de Economía Laboral
} 
años caía hasta el 62 por ciento.

Por otra parte, la tasa de desempleo femenina siempre ha sido superior a la de los hombres, a pesar de que durante los años de la última crisis económica se aproximaron mucho como consecuencia del notable aumento del desempleo masculino. A finales de 2017 las tasas eran 18,3 por ciento y 15 por ciento.

Las diferencias observadas entre los hombres y las mujeres en el empleo son un factor determinante de la brecha salarial, que se ha mantenido prácticamente inalterada a lo largo del tiempo. Por término medio, la brecha salarial que muestra que las mujeres cobran por hora trabajada casi un 15 por ciento menos que los hombres, o casi un 23 por ciento menos si se trata de ganancias anuales. Entre las razones que explican la brecha destacan aquellas que tienen que ver con la precariedad del empleo que afecta, sobre todo, a las mujeres. Y, por otro lado, por la existencia de segregación ocupacional.

Entre los determinantes más importantes de la precariedad laboral encontramos la elevada tasa de temporalidad y la creciente parcialidad en el empleo asalariado. Antes de 2007, las mujeres tenían una tasa de temporalidad que afectaba a más de un tercio de la población femenina asalariada; tras la caída de la temporalidad durante la crisis, la tasa se situó por debajo del 25 por ciento, pero con la recuperación ha vuelto a tener una tendencia creciente, situándose en 2017 en el 28 por ciento. Al mismo tiempo, la tasa de parcialidad, que estaba situada por debajo del 25 por ciento para las mujeres, comenzó a crecer durante la crisis y en 2017 más de un cuarto de la población femenina ocupaba un empleo asalariado a tiempo parcial; en cambio, esta proporción estaba en torno al 8 por ciento en el caso de los hombres. La doble precarización debida a la temporalidad y la parcialidad afecta a un 10 por ciento de las mujeres y a un 4 por ciento de los hombres. Cuando se pregunta a los trabajadores a tiempo parcial el motivo por el que tienen empleos con jornadas reducidas, más de la mitad argumenta que el motivo no es otro que el no haber podido acceder a un empleo a tiempo completo. De ahí que en España predomine el empleo a tiempo parcial involuntario.

Al analizar los datos de los registros de contratos del SEPE se observa que el empleo que se ha creado mayoritariamente estos años ha sido de carácter temporal con una tendencia creciente a que sea también a tiempo parcial, sobre todo entre las mujeres.

Estos datos muestras que, además, las diferencias entre los hombres y las mujeres se producen sobre todo a partir de la edad adulta.

Otro elemento que tiene mucho que ver con el empleo y las

(C) Revista de Economía Laboral 
diferencias entre hombres y mujeres procede de la segregación ocupacional. Ellas se concentran en ocupaciones de baja cualificación, un 17 por ciento del empleo femenino corresponde a ocupaciones elementales, y el "techo de cristal" impide que accedan a puestos mejor retribuidos y de más responsabilidad. A pesar de que el porcentaje de mujeres en cargos directivos es ya el 37 por ciento y de que hay más mujeres que nunca en el mercado de trabajo y en la cúpula de las empresas, sigue habiendo barreras sociales y culturales que impiden la igualdad laboral entre hombres y mujeres.

Un buen ejemplo de estas barreras tiene que ver con la necesidad de conciliar la vida familiar y laboral. Muchas mujeres han de orientar su formación y carrera profesional hacia empleos que terminan frenando su acceso a puestos mejor remunerados o se ven ante la tesitura de tener que renunciar o retrasar la maternidad.

Laura Bengoa and Arantza Ugidos de la Universidad del País Vasco, estudian cómo se ven afectados el tiempo dedicado dentro y fuera del mercado de trabajo en función de cuál sea la jornada de trabajo de los miembros de un hogar y qué consecuencias tiene para el nivel de bienestar de todos ellos. El estudio muestra que existe una relación negativa entre trabajar a jornada partida y el tiempo que se pasa en familia y con los hijos, dos actividades que mejoran la relación entre los miembros de la familia y mejoran el bienestar de los niños. Los padres pasan más tiempo con sus hijos cuando su cónyuge tiene un empleo a jornada partida o trabaja de tarde. El efecto negativo de la jornada partida o de trabajar de tarde es menor para las mujeres que para los hombres. Justo lo contrario es lo que se observa con el tiempo personal no familiar.

Nuria Legazpe y María A. Davia de la Universidad de Castilla La Mancha, analizan los patrones de participación laboral de madres y padres de niños pequeños y su vinculación con la demanda de cuidados formales e informales a lo largo del último cambio de ciclo en España. El estudio realizado confirma la relación positiva entre la demanda de cuidados formales e informales y la participación laboral de madres y padres. Otro de los efectos negativos de la crisis económica fue que esta relación se hizo más débil en el caso de las madres y más significativa en el de los padres, lo que pudiera indicar un cierto grado de implicación de los padres que perdieron el empleo durante la crisis en los cuidados de sus hijos pequeños, sustituyendo, esencialmente, a los cuidadores informales.

Diego Dueñas Fernández, de la Universidad de Alcalá, y Almudena Moreno Mínguez de la Universidad de Valladolid estudian en su trabajo las relaciones existentes entre la presencia de hijos en un 
hogar y el tiempo dedicado al empleo de las mujeres. Los resultados obtenidos mediante el análisis de corte transversal o a través de del uso de cohortes ficticias llevan a los autores a concluir que la maternidad condiciona y altera el tiempo dedicado al trabajo de las mujeres, las cuales deben reducir su dedicación laboral para aumentar su dedicación familiar.

Reyna Elizabeth Rodríguez Pérez y Myrna Limas Hernández, de Universidad Autónoma de Coahuila y la Universidad Autónoma de Ciudad Juárez respectivamente, analizan el aumento de la presencia de las mujeres en el mercado de trabajo a través de la revisión de algunas teorías y estudios empíricos que intentan explicar el tratamiento desigual que reciben las mujeres en el mercado laboral. Los principales resultados ponen una vez más de manifiesto que las mujeres son relegadas en ocupaciones de menor nivel que además, son consideradas propias o "adecuadas" para ellas.

Sergio Torrejón Pérez de la Universidad Complutense, elabora un índice para medir la vulnerabilidad del empleo con el objetivo de determinar cuáles son las ocupaciones y los trabajadores que se encuentran en una situación más vulnerable por estar empleados en puestos de trabajos relacionados con tareas consideradas más fáciles de remplazar debido al impacto del cambio tecnológico y los cambios en el comercio internacional. Además, se trata de identificar si son los hombres o las mujeres quienes se verán más afectados por este riesgo.

Albany Aguilera Fernández y David Castro Lugo de la Universidad Autónoma de Coahuila trasladan hasta México el estudio de la desigualdad laboral, centrándolo en las áreas urbanas del país. Utilizan un índice de desigualdad laboral que considera variables relacionadas con la participación laboral, ocupación, desempleo, remuneraciones, informalidad, segregación sectorial y condiciones laborales que les permite concluir que la desigualdad entre hombres y mujeres es un fenómeno que se ha mantenido persistente.

(C) Revista de Economía Laboral 